Bangladesh J. Bot. 50(3): 775-780, 2021 (September) SpecialＤOI: https://doi.org/10.3329/bjb.v50i5.56428

\title{
EFFECTS OF DIFFERENT HYDROGEN CONCENTRATIONS ON RHIZOSPHERE BACTERIA OF SOYBEAN (GLYCINE MAX L.)
}

\author{
Lin Wang, Bei Liu, Haiyan Li, Xiaoping Liang, Lulu Li ${ }^{1}$, \\ ZhiYING LI ${ }^{1}$ AND WENXIA HaN* \\ Department of Medical Technology, Xi'an Medical University, \\ Xi'an 710021, Shaanxi, China
}

Keywords: Rhizosphere, Hydrogen-oxidizing bacteria, Growth promotion, Bacterial diversity, Soybean

\begin{abstract}
Effects of hydrogen concentrations on the diversity and changes of bacterial community structure, soybean rhizosphere soil samples were investigated. The high-throughput sequencing technology of illumina was adopted to determine the bacterial 16S rRNA V3+V4 region series. Data processing such as splicing, filtering, removing chimeric sequences, and cluster analysis was then performed based on the raw data, and the tax was annotated with OTU. When the air-treated soil samples were compared with the ones treated with hydrogen at a concentration of $1100 \mathrm{ppm}$ and $1300 \mathrm{ppm}$, the abundance of Proteobacteria increased and Actinobacteria decreased for the later. In addition the Simpson index decreased and the Shannon index increased significantly for both hydrogen-treated samples. However, for the soil sample treated with 1500 ppm of hydrogen, the above-mentioned two indexes didnot vary obviously compared with the air-treated sample. The results demonstrated that the rhizosphere bacterial structure diversity of soybean was significantly increased after hydrogen treatment at the concentration of 1100 and $1300 \mathrm{ppm}$.
\end{abstract}

\section{Introduction}

The symbiosis of legume and rhizobia has the effect of nitrogen fixation. Generally, the soybean has a nitrogen fixation rate of 0 to $95 \%$ of the total nitrogen required by the plant under natural conditions (Maimaiti et al. 2007). However, the cultivation of soybean mainly relies on inorganic fertilizers to increase its yield. The rhizosphere of plant sustains a complex microecological system, which can be colonized by a large variety of bacteria (Orlando et al. 2007). Rhizosphere bacteria play important roles in element cycling in soil ecosystems, and are of very importance to plant health and soil fertility (Li et al. 2016). Changes in rhizosphere microbial community structure have an important impact on the circulation of matter and energy in the soil, the decomposition and synthesis of organic matter (Constant et al. 2010). Each plant species has a significant effect on the rhizosphere bacterial community structure due to the differences in root exudation, and it is thought that it may select own specific microbial populations in its rhizosphere (Farina et al. 2012). Bacteria are the most abundant organisms that reside in rhizosphere.

Leguminous plants produce hydrogen gas as a by-product during the process of nitrogen fixation (Osborne et al. 2010). However, for the other legumes, the uptake hydrogenase is absent and no hydrogen gas can be detected (Zhang et al. 2009). This phenomenon has indicated that hydrogen gas is absorbed by soil microbes called hydrogen-oxidizing bacteria (Irvine et al. 2004). Recent research now suggests that soil fertilization by hydrogen $\left(\mathrm{H}_{2}\right)$ gas may also be involved in enhancing the growth of both the legume and subsequent crops (Dong et al. 2003). That is, hydrogen may change the microbial population in the rhizosphere of plants (Irvine et al. 2004). Previous research has shown that $\mathrm{H}_{2}$ affects microbe-microbe interactions in soil in

*Author for correspondence: <w.w.wang@163.com>. ${ }^{1}$ Key Laboratory of Resource Biology and Biotechnology in Western China, Ministry of Education, Northwest University, Xi'an 710069, Shaanxi, China. 
addition to indirect effects on bacterial communities; and 958 bacterial ribotypes distributed among various taxonomic groups was influenced by $\mathrm{H}_{2}$ exposure (Uratsu et al. 1982). However, reports on the effects of different hydrogen concentrations on the rhizosphere microbial structure of legumes are not sufficient. Thus in the present study illumina high-throughput sequencing was used to investigate the microbial communities of the raw and soybean rhizosphere soil treated with three hydrogen concentrations on the basis of the PCR of the bacterial 16S rDNA gene.

\section{Materials and Methods}

The soil samples used in the experiment were collected in the soybean field of Xianyang City, Shaanxi Province, and 5 sampling points were selected in the soybean field by S-type sampling method. According to the shake-off method of Riley (Riley D et al. 1969), the soil on the soybean rhizosphere was brushed with a sterile brush. The five soybean soils were combined into one sample, and the sieve was evenly divided into two by a $2 \mathrm{~mm}$ sieve (George et al. 1995). These samples were placed in a sample bag and stored in a refrigerator at $-20^{\circ} \mathrm{C}$ for analysis. Three samples were treated with the hydrogen treatment equipment according to the method described by Dong and Layzell (2001). The instrumental indices of the dual integrated regulators that regulate the gas cycle are 12,18 , and 24 , respectively, to produce different concentrations of hydrogen, which are mixed with the incoming air to form a hydrogen-containing mixture (Smit et al. 2001). The conditions for setting the gas chromatograph are TG-BOND Msieve 5A GC column temperature: $50^{\circ} \mathrm{C}$, TCD detector temperature: $200^{\circ} \mathrm{C}$, split ratio: $15: 1$. Under these conditions, the hydrogen concentration produced by the gas circulation culture system is 1100 ppm (YA), 1300 ppm (YB) and 1500 ppm (YC), respectively. Three repetitions per sample were maintained and CK was treated as a control group (Bloem et al. 1995).

Soil total DNA was extracted using a PowerSoil® DNA Isolation Kit (Mo Bio Laboratories Inc., Carlsbad, CA, USA). After 1.8\% agarose gel electrophoresis, the concentration was measured by a nucleic acid quantitative spectrophotometer (nanodrop, USA) and stored in a refrigerator at $20^{\circ} \mathrm{C}$.

Using the extracted total DNA as a template, 336F (5'-ACT CCT ACG GGA GGC AGC A-3') and 806R (5'-GGA CTA CHVGGG TWT CTAAT-3') were used as primers to amplify the bacterial $16 \mathrm{~S}$ rRNA V3. +V4 area (Zhang et al. 2015). The total PCR reaction system is $50 \mu$ l, containing $0.2 \mu \mathrm{l}$ of Q5 high-fidelity DNA polymerase (New England BioLabs), $10 \mu \mathrm{l}$ of the buffer, and $10 \mu \mathrm{l}$ of a high GC Enhancer (Constant et al. 2008). PCR amplification was conducted according to the following protocols: initial denaturation at $95^{\circ} \mathrm{C}$ for $5 \mathrm{~min}$, followed by 15 cycles. Each cycle involved denaturation at $95^{\circ} \mathrm{C}$ for $1 \mathrm{~min}$, annealing at $50^{\circ} \mathrm{C}$ for $1 \mathrm{~min}$, and extension at $72^{\circ} \mathrm{C}$ for 1 min, with a final elongation step at $72^{\circ} \mathrm{C}$ for $7 \mathrm{~min}$ (Quail et al. 2012). The purified PCR products were quantified with Nanodrop 2000 Illumina high-throughput sequencing conducted at Biomarker Technologies (Beijing, China).

After the sequencing was completed, the sequenced fragments were spliced by overlap using FLASH v1.2.7 software, and the spliced sequence was obtained (Greening et al. 2014). Then, the original data were filtered using Trimmomatic v0.33 software to obtain the filtered data. The Uchime algorithm in the Mothur software was used to remove the chimeric sequence to obtain a high-quality Tags sequence (Schloss et al. 2009, Edgar et al. 2011). The sequence was clustered by 97\% similarity using UCLUST in the QIIME software to obtain the OUT classification data of the sample (Wang et al. 2012). Evaluation of sample Alpha diversity index using Mothur (version v.1.30) software The Bate diversity analysis was also performed using the QIIME software to compare the differences in the diversity of species for various species. The alpha diversity index was used to study the diversity of bacterial community structure. 


\section{Results and Discussion}

Alpha diversity reflects the richness and diversity of individual sample species. OTU Coverage, the higher the value, the higher the probability that the species in the sample will be measured. The coverage of each sample was greater than $99 \%$, indicating that the information of microbial species was fully reflected (Table 1). The larger the Shannon index value, the smaller the Simpson index value, indicating the higher species diversity of the sample (Grice et al. 2009). For the soybean rhizosphere soil samples, the Simpson index of the YA group was the smallest, the Shannon index was the largest. But for the YC group, the Simpson index was the largest and the Shannon index was the smallest. The results showed that the higher hydrogen concentration causes the lower microbial diversity of soybean rhizosphere soil.

Table 1. Alpha diversity index statistics.

\begin{tabular}{lllll}
\hline Sample ID & OTU & Simpson & Shannon & Coverage \\
\hline CK1 & 1490 & 0.0129 & 6.0656 & 0.9989 \\
CK2 & 1483 & 0.0126 & 0.0352 & 0.999 \\
CK3 & 1491 & 0.0128 & 6.0548 & 0.9992 \\
\hline YA1 & 1493 & 0.0043 & 6.3461 & 0.9989 \\
YA2 & 1497 & 0.004 & 6.357 & 0.9986 \\
YA3 & 1490 & 0.0049 & 6.3479 & 0.9991 \\
\hline YB1 & 1500 & 0.0043 & 6.3731 & 0.9992 \\
YB2 & 1504 & 0.0047 & 6.3526 & 0.9996 \\
YB3 & 1503 & 0.0053 & 6.3518 & 0.9991 \\
\hline YC1 & 1499 & 0.0163 & 6.0125 & 0.9994 \\
YC2 & 1492 & 0.0156 & 6.0322 & 0.999 \\
YC3 & 1490 & 0.0139 & 6.0606 & 0.9991 \\
\hline
\end{tabular}

Species composition analysis reflects the community structure of the samples at different taxonomic levels. Figure 1 shows the community structure and classification comparison results at the gate level. The top ten bacteria were the species of Proteobacteria, Acidobacteria, Actinobacteria, Bacteroidetes, Chloroflexi, Gemmatimonadetes, Planctomycetes, Verrucomicrobia, Rokubacteria, and Nitrospirae. In the soil samples treated with different hydrogen concentrations, the bacterial flora distribution was different. As the hydrogen concentration increased, the abundance of Proteobacteria and Bacteroidetes decreased. They decreased from 42 and $9.3 \%$ in the YA sample to 34 and $6.9 \%$ in the YC sample, respectively and correspondingly. The abundance of Actinobacteria and Planctomycetes were increased. They increased from 11 and $1.5 \%$ in YA samples to 23 and $1.9 \%$ in YC samples, respectively and correspondingly.

Principal coordinates analysis (PCoA) (Mouradi et al. 2016) using variance decomposition, the differences of multiple sets of data are reflected on the two-dimensional graph, and the coordinate axes take two eigenvalues that can reflect the variance maximally. The closer the distance between the two samples, the more similar the species composition of the two samples. In the PCoA plot, the different generations represent different groups, and the horizontal and vertical coordinates are the two eigenvalues that cause the largest difference between the samples. The extent of the effect of the sample is expressed as a percentage. 


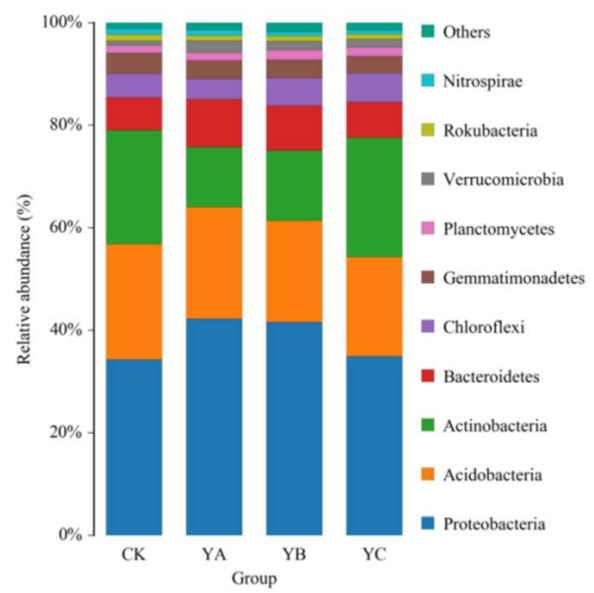

Fig. 1. Distribution of sample communities at phylum level.

The PCoA results of this experiment are presented in Fig. 2. The duplicate group samples were relatively clustered together with inapparent differences, which indicate its feasibility of the repeat group setting. The effect of $\mathrm{PC} 1$ on sample differences was $40.23 \%$ and caused the samples $\mathrm{CK}$ and $\mathrm{YB}$ to be grouped together. It promoted the $\mathrm{YB}$ group significantly but inhibited the $\mathrm{CK}$ group. The effect of PC2 on sample differences was $18.94 \%$. It has the obvious promoting effect on the sample YC group, the obvious inhibitory effect on the sample $\mathrm{CK}$, and no obvious effect on the sample YA and YB. Therefore, the experiment results showed that as the hydrogen treatment was gradually strengthened, the species composition varied greatly between samples. It indicates that the hydrogen concentration had a significant effect on the bacterial species composition of the soybean rhizosphere.

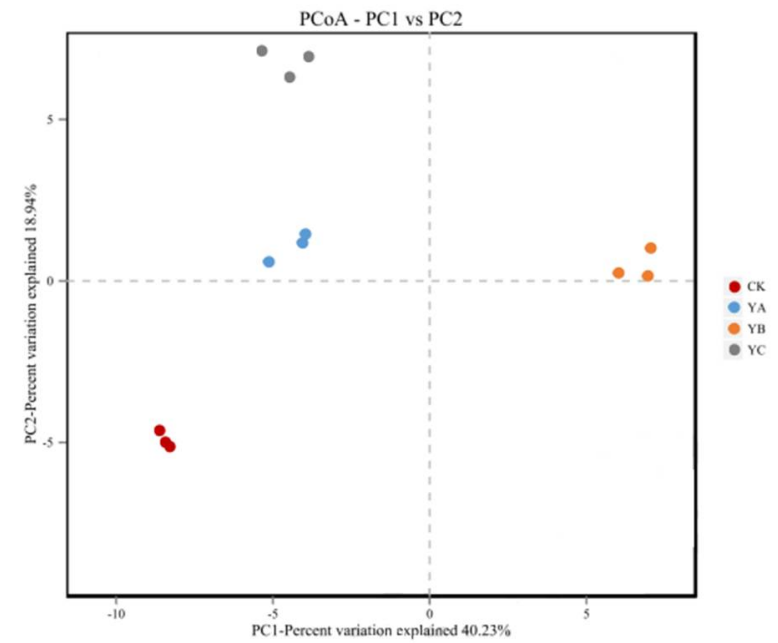

Fig. 2. Principal coordinates analysis.

The clone library showed that the division of Proteobacteria, Acidobacteria and Actinobacteria dominated the rhizosphere samples of soybean. It was also found that these three groups dominated the root samples treated with different hydrogen concentrations, accounting for 
more than $80 \%$ of the bacterial sequence. These results are consistent with previous studies (Stursová et al. 2012, Piche-Choquette et al. 2016). When the hydrogen concentration was 1500 $\mathrm{ppm}$, the community structure of soybean rhizosphere soil samples has no significant difference from that of the non-hydrogen treated control group (CK). Compared with the treatment group with a hydrogen concentration of $1100 \mathrm{ppm}$ and $1300 \mathrm{ppm}$, the abundance of its Proteobacteria decreased, while the abundance of its Actinobacteria increased. This observation is similar to previous reports (Li et al. 2018). The probable cause is that $1500 \mathrm{ppm}$ is higher than the threshold for oxidizing hydrogen in the Proteobacteria, and Proteobacteria cannot use a hydrogen concentration above the threshold to cause abundance reduction (Duan et al. 2013).

Thus, the hydrogen concentration of 1100 and $1300 \mathrm{ppm}$ changed the bacterial diversity of the soybean rhizosphere soil significantly whereas 1500 ppm hydrogen showed a little effect.

\section{Acknowledgements}

This study was supported by the Special Research Project of the Education Department of Shaanxi Province (Grant No. 21JK0885), the Supporting Funds of Xi'an Medical University (2018PT73), the Opening Foundation of Shaanxi Provincial Key Laboratory of Brain Disease Prevention and Treatment (Grant No. 20NBZD03), the Youth Innovation Team of Shaanxi Universities (Grant No. 90 [2019]) and National Science and Technology Gold Cultivation Project (Grant No.2017GJFY15). The authors thank Biomarker Technologies for their excellent technical assistance.

\section{References}

Bloem J, Veninga M and Sheppard J 1995. Fully automated determination of soil bacterium numbers, cell volumes, and frequencies of dividing cells by confocal laser scanning microscopy and image analysis. Appl. Environ. Microbiol. 61: 926-936.

Constant P, Chowdhury SP, Pratscher J and Conrad R 2010. Streptomycetes contributing to atmospheric molecular hydrogen soil uptake are widespread and encode a putative high-affinity [NiFe]-hydrogenase. Environ. Microbiol. 12: 821-829.

Constant P, Poissant L and Villemur R 2008. Isolation of Streptomyces sp. PCB7, the first microorganism demonstrating high-affinity uptake of tropospheric H2. Isme J 2: 1066.

Dong Z, Layzell DB 2001. H2 oxidation, O2 uptake and CO2fixation in hydrogen treated soils. Plant Soil 229: $1-12$.

Dong Z, Wu L, Kettlewell B, Caldwell CD and Layzell DB 2003. Hydrogen fertilization of soils-is this a benefit of legumes in rotation? Plant Cell Environ. 26:1875-1879.

Duan Q, Liao TW 2013. A new age-based replenishment policy for supply chain inventory optimization of highly perishable products. Int. J. Pro. Econ. 145: 658-671.

Edgar RC, Haas BJ, Clemente JC and Quince 2011. UCHIME improves sensitivity and speed of chimera detection. Bioinformatics 27: 2194-2200.

Farina R, Beneduzi A, Ambrosini A, de Campos SB, Lisboa BB, Wendisch V, Vargas LK and Passaglia LMP 2012. Diversity of plant growth-promoting rhizobacteria communities associated with the stages of canola growth. Appl. Soil Ecol. 55(1): 44-52.

George E, Marschner H and Jakobsen I 1995. Role of arbuscular mycorrhizal fungi in uptake of phosphorus and nitro-gen from soil. Crit Rev. Biotech. 15: 257-270.

Greening C, Berney M, Hards K, Cook GM and Conrad R 2014. A soil actinobacterium scavenges atmospheric H2 using two membrane-associated, oxygen-dependent [NiFe] hydrogenases. Proc Natl Acad Sci USA 111: 4257-4261.

Grice EA, Kong HH, Conlan S, Grice EA, Deming CB, Davis J, Young AC, Bouffard GG, Blakesley RW, Murray PR and Green ED 2009. Topographical and temporal diversity of the human skin microbiome. Science 324:1190-1192. 
Xiang H 2014. Assessment of soil bacterial community structure changes in response to hydrogen gas released by $\mathrm{N}_{2}$-fixing nodules. Int. J. Plant Soil Sci. 3: 47-61.

Irvine P, Smith M and Dong Z 2004. Hydrogen fertilizer: bacteria or fungi? Acta Hort. 631: 239-242.

Li L, Wei LN and Cui JQ 2016. Effects of funneliformismosseae on the arbuscular mycorrhizal fungal community structure of root systems at the branching stage of continuous soybean cropping. Mycosystema, 35: 882-891.

Li Z, Liu X, Liu R, Li L, Wang L and Wang W 2018. Insight into bacterial community diversity and monthly fluctuations of Medicagosativa rhizosphere soil in response to hydrogen gas using Illumina HighThroughput Sequencing. Curr Microbiol. 75(12): 626-1633.

Maimaiti J, Zhang Y, Yang J, Cen YP, Layzell DB and Dong Z 2007. Isolation and characterization of hydrogen-oxidizing bacteria induced following exposure of soil to hydrogen gas and their impact on plant growth. Environ Microbiol. 9(2): 435-444.

Mouradi M, Farissi M, Bouizgaren A, Makoudi B, Kabbadj A, Very AA, Sentenac H, Qaddourya A and Ghoulam C 2016. Effects of water deficit on growth, nodulation and physiological and biochemical processes in Medicago sativa-rhizobia symbiotic association. Arid Land Res Mana 30: 193-208.

Orlando J, Chavez M, Bravo L, Guevara, R and Margarita Carú 2007. Effect of Colletiahystrix (Clos), a pioneer actinorhizal plant from the Chilean matorral, on the genetic and potential metabolic diversity of the soil bacterial community. Soil Biol. Biochem. 39: 2769-2776.

Osborne CA, Peoples MB and Janssen PH 2010. Detection of a reproducible, single-member shift in soil bacterial communities exposed to low levels of hydrogen. Appl. Environ. Microbiol. 76: 1471-1479.

Piche-Choquette S, Tremblay J, Tringe SG and Constant P 2016. H2-saturation of high affinity H2-oxidizing bacteria alters the ecological niche of soil microorganisms unevenly among taxo-nomic groups. Peer. J. 4: e1782.

Quail MA, Smith M and Coupland P 2012. A tale of three next generation sequencing platforms: comparison of ion torrent, pacific biosciences and illumina MiSeq sequencers. Genetics, 13: 34-41

Riley D and Barber SA 1969. Bicarbonate Accumulation and pH Changes at the Soybean (Glycine max (L.) Merr.) Root-Soil Interface. Soil Science Society of America Journal 33(6): 905.

Schloss PD, Westcott SL, Ryabin T, Hall, J. R, Hartmann, M, EB Hollister, Lesniewski RA, Oakley BB, Parks DH and Robinson CJ 2009. Introducing mothur: Open-source, platform-independent, communitysupported software for describing and comparing microbial communities. Appl. Environ. Microb. 75:7537.

Smit E, Leeflang P, Gommans S, Broek JVD and Wernars K 2001. Diversity and seasonal fluctuations of the dominant members of the bacterial soil community in a wheat field as determined by cultivation and molecular methods. Appl. Environ. Microb. 67: 2284-2291.

Stursová M, Zifčáková L, Leigh MB, Burgess R and Baldrian P 2012. Cellulose utilization in forest litter and soil: identification of bacterial and fungal decomposers. Fems Microbiol. Ecol. 80: 735-746

Uratsu SL, Keyser HH, Weber DF and Lim ST 1982. Hydrogen up-take (HUP) activity of Rhizobium japonicum from major U.S. soybean production areas. Crop Sci. 22: 600-602

Wang Y, Sheng H, He Y, Wu JY, Jiang YX, Tam FY and Zhou HW 2012. Comparison of the levels of bacterial diversity in freshwater, intertidal wetland, and marine sediments by using millions of illumina tags. Appl. Environ. Microb. 78: 8264-8271.

Zhang X, Tian X, Ma L, Feng B and Liu Q, 2015. Biodiversity of the symbiotic bacteria associated with toxic marine dinoflagellate Alexan-drium tamarense. J. Biosci. Medicines 03: 23-28.

Zhang Y, He X and Dong Z 2009. Effect of hydrogen on soil bacterial community structure in two soils as determined by terminal restriction fragment length polymorphism. Plant \& Soil 320(1-2): 295-305. 